\title{
Identifying New Isatin Derivatives with GSK-3 $\beta$ Inhibition Capacity through Molecular Docking and Bioassays
}

\author{
Karolinni B. Britto, ${ }^{a}$ Carla S. Francisco, ${ }^{b}$ Débora Ferreira, ${ }^{c}$ Bárbara J. P. Borges, ${ }^{a}$ \\ Raphael Conti,,$^{b}$ Demetrius Profeti, ${ }^{\circledR d}$ Ligia R. Rodrigues, ${ }^{\oplus c}{ }^{\circ}$ Valdemar Lacerda Jr., ${ }^{\circ b}$ \\ Pedro A. B. Morais*,\#,d and Warley S. Borges ${ }^{\circledR *, \#, a, b}$
}

aPrograma de Pós-Graduação em Ciências Farmacêuticas, Universidade Federal do Espírito Santo, 29075-910 Vitória-ES, Brazil

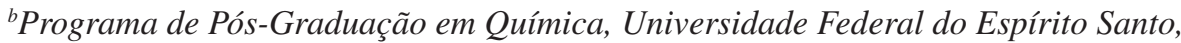
29075-910 Vitória-ES, Brazil

${ }^{c}$ Centre of Biological Engineering, University of Minho, Campus de Gualtar, 4710-057 Braga, Portugal

${ }^{d}$ Centro de Ciências Exatas, Naturais e da Saúde, Universidade Federal do Espírito Santo, 29500-000 Alegre-ES, Brazil

\begin{abstract}
The semi-synthesis of 11 isatin derivatives was achieved through bimolecular nucleophilic substitution and click chemistry. Seven new compounds were obtained. All chemical structures were determined by infrared spectroscopy (IR), nuclear magnetic resonance spectrometry (NMR) and high-resolution mass spectrometry (HRMS) data. These derivatives were evaluated for their anti-GSK-3 $\beta$ activity and all isatin derivatives ( $N$-alkyl and 1,2,3-triazolic) exhibited strong inhibitory activity, with $\mathbf{2 b}$ and $\mathbf{4 h}$ exhibiting remarkable potency. In addition, docking studies were performed with $\mathbf{2} \mathbf{b}$ and $\mathbf{2 e}$ models to unravel the molecular mechanism underlying the polar interactions on the GSK-3 $\beta$ ATP-binding site.
\end{abstract}

Keywords: isatin derivatives, GSK-3 $\beta$, molecular docking

\section{Introduction}

Isatin ( $1 H$-indole-2,3-dione), first synthesized by Erdmann $^{1}$ and Laurent ${ }^{2}$ in 1840 , results from the reaction of indigo with nitric and chromium acids. This molecule has been isolated from Bufo frogs, fungi and marine molluscs. ${ }^{3}$ In plants, the isatin alkaloid was found in Strobilanthes cusia (Nees), Isatis tinctoria L., ${ }^{4}$ Couroupita guianesis Aubl..$^{5}$ and Calanthe discolor. ${ }^{6}$ These plants have an ethnic relevance in traditional Chinese therapeutics. ${ }^{3}$

The Sandmeyer method ${ }^{7}$ is a broadly used procedure to synthesize isatin. This method uses a mixture of chloral hydrate, aniline (or its hydrochloride salt), hydroxylamine and hydrochloric acid in aqueous medium saturated with heated sodium sulfate. This results in isonitrosoacetanilide

*e-mail: pedro.morais@ufes.br; warley.borges@ufes.br

"Both authors contributed equally to this work. sulfate, which is then isolated and treated with sulfuric acid, to ultimately generate isatin by cyclization. ${ }^{7}$ However, other processes for the synthesis of isatin are described in the literature. ${ }^{8}$

Isatin is still considered to be a promising agent during the planning of new drugs given its low production costs and its chemical structure. The possibility to introduce diverse chemical modifications to isatin enables the synthesis of new substances that have very interesting and unexplored biological activities. ${ }^{9}$

Isatin and its derivatives are widely related to their diverse biological activities that include antifungal, ${ }^{10,11}$ antiinflammatory and analgesic; ${ }^{12}$ antimicrobial $;{ }^{13}$ antiviral $;{ }^{14}$ antiasthmatic; ${ }^{15}$ antioxidant; $;{ }^{16}$ herbicide; $;{ }^{17}, 18$ anticancer, ${ }^{19}$ among others.

The drug sunitinib, approved in 2006 by the FDA (Food and Drug Administration), highlights the potential of isatin structures in the development of new treatments for renal, gastrointestinal ${ }^{20}$ and pancreatic cancers. ${ }^{21}$ 
The inhibition of protein kinases through an important indole-based compound mechanism underlies the anticancer activity of isatins. Protein kinases are responsible for regulating intra and extracellular pathways that control growth, differentiation and cell death. ${ }^{3}$ Krishnegowda et al. ${ }^{19}$ showed that some $\mathrm{N}$-alkylated isatins containing bromine atoms in positions 5 and 7 had cytotoxic activity against human melanoma (UACC903), breast (MCF-7), lung (A549), and colon (HT29) cancers. Singh et at. ${ }^{22}$ synthesized 1,2,3-triazole-linked isatin conjugates and evaluated their anticancer activities against four human cancer cell lines, namely A549 (lung), PC-3 (prostate), THP-1 (leukemia) and Caco2 (colon). The most promising results were obtained for the THP-1 cells.

Although the exact mechanism of action of these compounds remains unclear, it has been suggested that they are able to inhibit glycogen synthase kinase 3 (GSK-3) in tumor cells and, consequently, cause tumor cells death. ${ }^{23,24}$

A surprising (though underestimated) protein, GSK-3 is one of the kinases involved in phosphorylation and inactivation of glycogen synthase..$^{25}$ The growing interest in this enzyme (whose action is constitutive and its substrates suffers pre-phosphorylation by kinases) has led to the discovery of GSK-3 $\alpha$ and GSK-3 $\beta$ isoforms with remarkable homology at the catalytic site and $\mathrm{N}$ and $\mathrm{C}$ termini differences. ${ }^{26}$ At the N-terminus, GSK-3 $\alpha$ has a 63-residue glycine-rich insertion. At the C-terminus, the sequence homology of the last 80 residues drops to $34 \%$. There is one additional GSK-3 $\beta$ isoform that contains a 13 amino acid insertion in the kinase domain. ${ }^{27} \mathrm{~A}$ primedsubstrate, attracting substrates to GSK-3, and kinase, related to phosphorylation of ones, essential domains have been characterized and in both isoforms GSK-3 $\alpha$ and GSK-3 $\beta$ a phosphorylation in Ser21 and Ser9 residues, respectively, is intrinsically needed for catalytic action. ${ }^{28}$

The GSK-3 $\beta$ is a 420-residue serine/threonine kinase. The first crystallographic structures of this enzyme were published in 2001 by Haar et al. ${ }^{29}$ These structures revealed a typical serine/threonine kinase fold with a small $\mathrm{N}$-terminal domain (residues 25 to 134) and a larger C-terminal domain (residues 135 to 380 ). Moreover, the $\mathrm{N}$-terminal portion was found to consist of seven antiparallel $\beta$-strands and the $\alpha-C$ helix. $\beta$-Strands two through six form an antiparallel $\beta$-barrel that is interrupted by the $\alpha-C$ helix (residues Arg96 to Met101) between $\beta$-strands five and six. The adenosine triphosphate (ATP) binding site is located at the interface of the $\mathrm{N}$-terminal and C-terminal domains and is enclosed by the glycinerich loop (residues 60 to 70) and the hinge (residues 134 to 139), comprising amino acids Ile62, Gly63, Asn64, Val70, Ala83, Lys85, Val110, Leu132, Asp133, Tyr134, Val135, Thr138, Arg141, Gln185, Leu188, Cys199 and Asp200.
The activation loop starts with the DFG (Asp-PheGly) motif (Asp200 to Gly202), finishes with the APE (Ala-Pro-Glu) motif (Ala224 to Glu226), and forms one border with the substrate-binding groove. The other edge is formed by the loop that connects $\beta$-strand five with the $\alpha-C$ helix. The final 55 residues after the kinase domain (residues 330 to 384) create a cluster of loops and helices that pack against the $\mathrm{C}$-terminus portion. The Protein Data Bank (PDB) contains information about 61 GSK-3 $\beta$ structures, nine each of apo-GSK-3 $\beta$ and GSK-3 $\beta$-peptide complexes and remainder of GSK-3 $\beta$ complexes with inhibitors in the ATP binding site. ${ }^{27}$

Human GSK-3 $\beta$ is a $46-47$ kilodalton $(\mathrm{kDa})$ protein presenting 433 amino acids in its structure and an activation loop portion that is pivotal for kinase activity due to the presence of phosphorylated residues. Ser9 and Tyr28 phosphorylation is a precondition for enzymatic action regulation since these residues control the gate of the substrate-binding site. ${ }^{30,31}$ Ser9 is an auto-inhibition site that inhibits the catalytic activity when phosphorylated. This phosphorylation site is disordered in GSK-3 $\beta$ crystals and not identified in crystallographic information. Tyr216 is the phosphorylation site in the activation loop. Phosphorylation of Tyr216 increases the catalytic activity of GSK-3 $\beta$. Threonine is absent in the loop of the primed-substrate domain in GSK-3 $\beta$, and recognition of the primed phosphorylation on substrates occurs at this site. Furthermore, due to absence of a threonine residue, following phosphorylation of the serine residue, Ser9, the GSK-3 $\beta$ N-terminal region binds to and blocks the catalytic site. ${ }^{29,32}$ Finally, there is an important similarity between enzyme and apoenzyme structures, and the GSK-3 $\beta$ protein crystallographic structure presented as a dimer with identical subunits.

In this work, we describe the synthesis of 11 isatin derivatives that were obtained in low to high yields. These compounds were characterized by means of nuclear magnetic resonance spectrometry (NMR), infrared spectroscopy (IR), and high-resolution mass spectrometry (HRMS). Additionally, characterization of the isatin derivatives was performed through enzymatic inhibition assays and docking simulations to obtain a better understanding of GSK-3 $\beta$ structural features and its interactions with staurosporine.

\section{Experimental}

\section{General experimental procedures}

Analytical grade solvents with purity higher than $99.5 \%$ were purchased from Synth (São Paulo, Brazil). Isatin, 
dimethylformamide (DMF), acetone azides, bromides, potassium carbonate, sodium ascorbate, copper sulfate, propargyl bromide solution ( $80 \mathrm{wt} . \%$ in toluene, containing $0.3 \%$ of magnesium as stabilizer) and deuterated solvents were purchased from Sigma-Aldrich (St. Louis, MO, USA). Microwave reactions were conducted using a CEM Discover Synthesis Unit (CEM Corp., Matthews, USA). The instrument consists of a continuous focused microwave power delivery system with operator-selectable power output from 0 to $300 \mathrm{~W}$. Reactions were performed in glass vessels (capacity $10 \mathrm{~mL}$ ) sealed with a septum. Column chromatography (CC) was carried out using silica gel 60 (70-230 mesh, Merck (Darmstadt, Germany)) and silica gel 60 ACC (6-35 $\mu \mathrm{m}$, Chromagel-SDS, Torrance, USA) as the stationary phase, and mixtures of hexane and ethyl acetate as the mobile phase. For thin layer chromatography (TLC), silica gel F254 was used as the stationary phase with plates of dimension $20 \mathrm{~cm} \times 20 \mathrm{~cm} \times 0.20 \mathrm{~mm}$. NMR (nuclear magnetic resonance) spectra were obtained with a Varian $400 \mathrm{MHz}$ instrument (Palo Alto, USA) using deuterated chloroform $\left(\mathrm{CDCl}_{3}\right)$ or deuterated dimethyl sulfoxide (DMSO- $d_{6}$ ) as solvent and tetramethylsilane (TMS) as the internal standard. The chemical shift $(\delta)$ is in ppm and $J$ values in hertz $(\mathrm{Hz})$. High resolution mass spectra were obtained with a Bruker Daltonics micrOTOF QII/ESI-TOF (Billerica, USA) by direct injection of the compound dissolved in methanol. This provided unambiguous molecular formula assignment for singly charged molecular ions, such as $[\mathrm{M}+\mathrm{H}]^{+}$or $[\mathrm{M}-\mathrm{H}]^{-}$and DBE (double bound equivalents) values. The infrared (IR) spectrum was recorded on a Bruker Tensor 27 FT-IR Spectrometer (Bremen, Germany), equipped with a Pike MIRacle attenuated total reflection (ATR) assembly with a ZnSe crystal, scanning from 4000 to $600 \mathrm{~cm}^{-1}$.

\section{Synthesis procedures}

\section{Synthesis of the compounds $\mathbf{2 a - 2 f}$}

Isatin (1) $(0.05 \mathrm{~g}, 0.35 \mathrm{mmol})$ and potassium carbonate $(0.05 \mathrm{~g}, 0.35 \mathrm{mmol})$ were added to a $50 \mathrm{~mL}$ round-bottom flask containing $4 \mathrm{~mL}$ of dimethylformamide (acetone in preparation of compound $\mathbf{2 c}$ ). The reaction solution was stirred at $0{ }^{\circ} \mathrm{C}$ for $20 \mathrm{~min}$. After this process, each bromide (a-e) $(0.51 \mathrm{mmol})$ was added separately to the reaction solution and the mixture was stirred at room temperature for $3 \mathrm{~h}$, leading to compounds $\mathbf{2 a - e}$ (Scheme 1).

The reaction solution was concentrated under reduced pressure. The compounds $\mathbf{2 a - c , e}$ were purified by column chromatography using silica gel 60 (70-230) mesh as the stationary phase and hexane:ethyl acetate $7: 3$ as the mobile phase. To purify compound $\mathbf{2 d}$, an extraction with ethyl acetate was used.

The obtained percentages and quantitative yields of the compounds $2 \mathrm{a}-\mathbf{e}$ were $70.29 \%(0.087 \mathrm{~g}, 0.23 \mathrm{mmol})$, $29.35 \%$ ( $0.027 \mathrm{~g}, 0.09 \mathrm{mmol}), 10.40 \%$ ( $0.013 \mathrm{~g}, 0.03 \mathrm{mmol})$ and $55.13 \%$ (0.048 g, $0.18 \mathrm{mmol})$, respectively.

To synthesize the compound $\mathbf{2 f}$, isatin (1) (1 g, $6.7 \mathrm{mmol})$ and potassium carbonate $(0.92 \mathrm{~g}, 6.7 \mathrm{mmol})$ were added to a $100 \mathrm{~mL}$ round-bottom flask containing $20 \mathrm{~mL}$ of dimethylformamide. The reaction solution was stirred in ice bath for $20 \mathrm{~min}$. After this process, propargyl bromide (f) $(8.37 \mathrm{mmol})$ was slowly poured into the reaction solution, and the solution was stirred at room temperature for $3 \mathrm{~h}$. Afterwards, the reaction was concentrated under reduced pressure, resuspended in ethyl acetate and partitioned with water 3 times. The organic phase was concentrated under reduced pressure leading to compound $\mathbf{2 f}$ (Scheme 1).

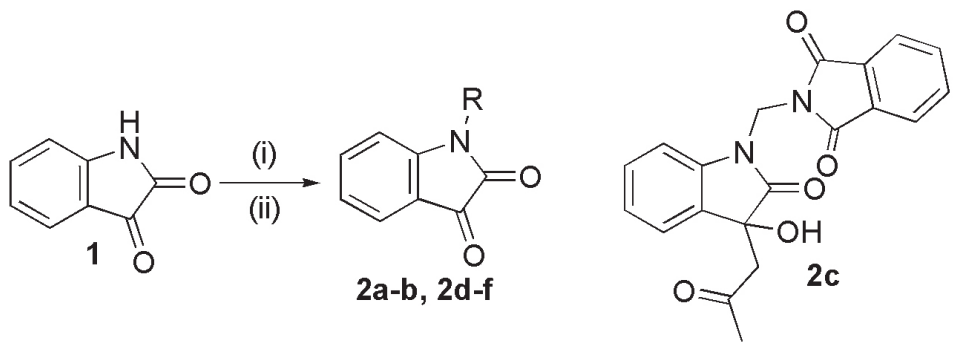<smiles>[R]#CCc1ccc2c(c1)C(=O)c1ccccc1C2=O</smiles>

a<smiles>O=[N+]([O-])c1ccc([N+](=O)[O-])cc1</smiles>

b<smiles>CCN1C(=O)c2ccccc2C1=O</smiles>

c<smiles>CCCc1ccc(F)cc1</smiles>

d<smiles>CC(C)(C)Cc1ccccc1CS(=O)(=O)c1ccccc1</smiles>

e

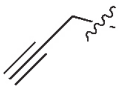

$\mathbf{f}$

Scheme 1. (i) Potassium carbonate, dimethylformamide or acetone; (ii) commercial bromide, r.t. 


\section{Synthesis of the compounds k-I}

$\mathrm{NaN}_{3}$ (1.5 equiv.) dissolved in water was slowly added to a $100 \mathrm{~mL}$ round-bottom flask containing bromide $(\mathbf{e}$, g) (1 equiv.) in $10 \mathrm{~mL}$ of acetone at $0{ }^{\circ} \mathrm{C}$. The reaction solution was stirred at room temperature for $3 \mathrm{~h}$. After this process, acetone was removed under vacuum and the organic material was extracted with ethyl acetate that was further evaporated under reduced pressure generating the compounds $\mathbf{k}$ and $\mathbf{I}$ (Scheme 2). Their chemical structures were determined by IR and HRMS.<smiles>O=S(=O)(Cc1ccccc1CBr)c1ccccc1</smiles>

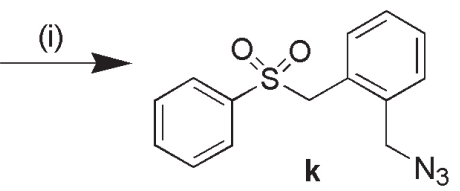

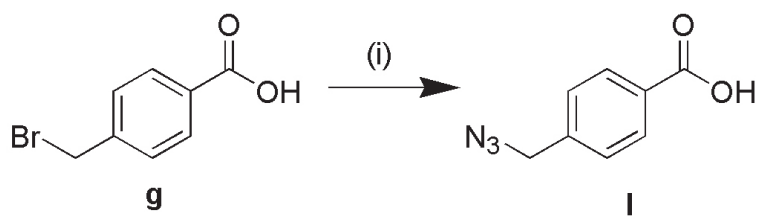

Scheme 2. (i) Sodium azide, acetone, r.t.

\section{Synthesis of the compounds $4 \mathrm{~h}-4 \mathrm{I}$}

In a $10 \mathrm{~mL}$ glass tube, the compound $2 \mathrm{f}(0.1 \mathrm{~g}$, $0.5 \mathrm{mmol}$ ), the obtained or the commercial azides ( $2.1 \mathrm{mmol})$, sodium ascorbate $(0.05 \mathrm{mmol}), 150 \mu \mathrm{L}$ of a $0.1 \mathrm{M}$ solution of copper sulfate, $2 \mathrm{~mL}$ of DMF, and a magnetic stir bar were combined. The vessel was sealed with a septum and placed into the microwave cavity. Microwave irradiation of $150 \mathrm{~W}$ was used; the temperature was ramped from room temperature to $70{ }^{\circ} \mathrm{C}$, and the reaction mixture was held at the final temperature for $15 \mathrm{~min}$. A minor modification was made to this procedure for compound $\mathbf{4 l}$, where the reaction mixture was held at $70{ }^{\circ} \mathrm{C}$ for only $5 \mathrm{~min}$ (Scheme 3 ). After this process, the DMF was removed under vacuum.

All compounds were purified by column chromatography using silica gel $60 \mathrm{ACC}(6-35 \mu \mathrm{m})$ as the stationary phase and hexane:ethyl acetate 9:1 (for compound $\mathbf{4 j}$ ), hexane:ethyl acetate 7:3 (for compounds $\mathbf{4 h}, \mathbf{4 i}$ and $\mathbf{4 k}$ ) and hexane:ethyl acetate 4:1 (for compound 4l) as the mobile phase.

Percentages and quantitative yields obtained for the compounds $4 \mathbf{h}-\mathbf{l}$ were $70.38 \%$ (0.141 g, $0.37 \mathrm{mmol})$, $17.66 \%$ (0.032 g, $0.09 \mathrm{mmol}), 24.12 \%$ (0.050 g, $0.12 \mathrm{mmol}$ ), $19.43 \%(0.012 \mathrm{~g}, 0.02 \mathrm{mmol})$ and $51.44 \%(0.030 \mathrm{~g}$, $0.08 \mathrm{mmol})$, respectively.

Compound 2a (1-(9,10-dioxo-9,10-dihydro-anthracen2-ylmethyl)-1 $H$-indole-2,3-dione)

It was obtained as orange powder in $70.29 \%$ yield $(0.087 \mathrm{~g}, 0.23 \mathrm{mmol})$. TLC: retention factor $(\mathrm{Rf})=0.42$ (hexane:ethyl acetate $7: 3 \mathrm{v} / \mathrm{v}$ ); IR (ATR) $v_{\max } / \mathrm{cm}^{-1} 2922$, 1730, 1673, 1605, 1292, 706; ' ${ }^{1} \mathrm{H}$ NMR (400 MHz, $\mathrm{CDCl}_{3}$ ) $\delta$ 8.26-8.33 (m, 4H), 7.79-7.84 (m, 2H), $7.76(\mathrm{dd}, J$ 8.2, $1.9,1 \mathrm{H}), 7.66(\mathrm{dd}, J 7.8,0.8,1 \mathrm{H}), 7.50(\mathrm{td}, J 7.8,1.2,1 \mathrm{H})$, $7.13(\mathrm{td}, J 7.8,0.8,1 \mathrm{H}), 6.76(\mathrm{brd}, J 7.8,1 \mathrm{H}), 5.10(\mathrm{~s}, 2 \mathrm{H})$; ${ }^{13} \mathrm{C}$ NMR (100 MHz, $\left.\mathrm{CDCl}_{3}\right) \delta 182.7(\mathrm{CO}), 182.5(\mathrm{CO})$, $182.4(\mathrm{CO}), 158.3(\mathrm{CO}), 150.1(\mathrm{C}), 141.3(\mathrm{C}), 138.5(\mathrm{CH})$, $134.4(2 \mathrm{C}), 134.3(\mathrm{C}), 134.0(\mathrm{CH}), 133.3(2 \mathrm{CH}), 133.2(\mathrm{C})$, $132.7(\mathrm{CH}), 128.4(\mathrm{CH}), 127.3(2 \mathrm{CH}), 125.8(\mathrm{CH}), 124.3$

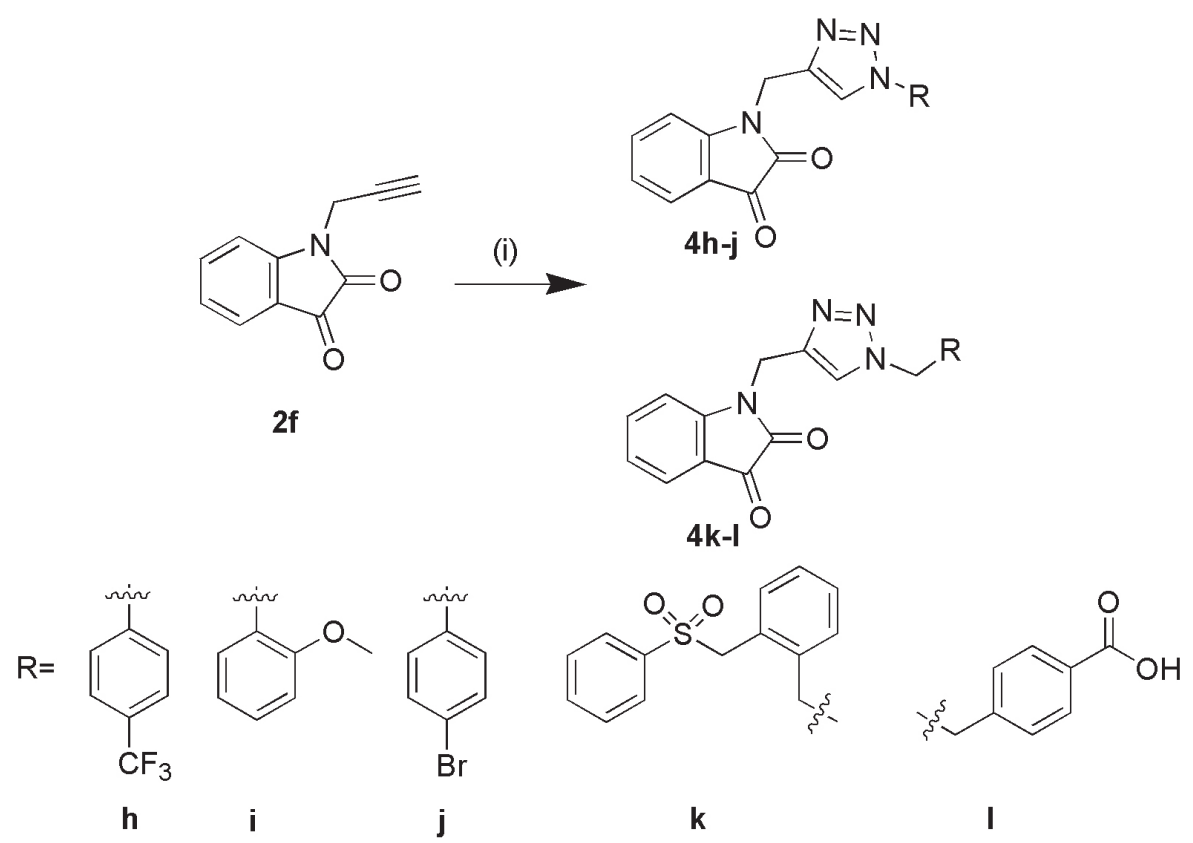

Scheme 3. (i) Azide compounds, sodium ascorbate, copper sulfate, dimethylformamide, $70{ }^{\circ} \mathrm{C}$. 
$(\mathrm{CH}), 117.8(\mathrm{CH}), 110.6(\mathrm{CH}), 43.8\left(\mathrm{CH}_{2}\right)$; high-resolution electrospray ionisation mass spectrometry (HRESIMS) $m / z$, calcd. for $\mathrm{C}_{23} \mathrm{H}_{14} \mathrm{NO}_{4}{ }^{+}[\mathrm{M}+\mathrm{H}]^{+}: 368.0917$, found: 368.0817 .

\section{Compound 2b (1-(4-nitro-benzyl)-1H-indole-2,3-dione)}

It was obtained as yellow powder in $29.35 \%$ yield $(0.027 \mathrm{~g}, 0.09 \mathrm{mmol})$. TLC: $\mathrm{Rf}=0.39$ (hexane: ethyl acetate $7: 3 \mathrm{v} / \mathrm{v})$; IR (ATR) $v_{\max } / \mathrm{cm}^{-1} 2925,1733,1372,759 ;{ }^{1} \mathrm{H}$ and ${ }^{13} \mathrm{C}$ NMR data were compared with the literature. ${ }^{33-35}$ HRESIMS $m / z$, calcd. for $\mathrm{C}_{15} \mathrm{H}_{11} \mathrm{~N}_{2} \mathrm{O}_{4}^{+}[\mathrm{M}+\mathrm{H}]^{+}: 283.0713$, found: 283.0713; calcd. for $\mathrm{C}_{15} \mathrm{H}_{10} \mathrm{~N}_{2} \mathrm{NaO}_{4}{ }^{+}[\mathrm{M}+\mathrm{Na}]^{+}$: 305.0533, found: 305.0538 .

Compound 2c (2-[3-hydroxy-2-oxo-3-(2-oxo-propyl)2,3-dihydro-indol-1-ylmethyl]-isoindole-1,3-dione)

It was obtained as orange powder in $10.40 \%$ yield ( $0.013 \mathrm{~g}, 0.03 \mathrm{mmol})$. TLC: $\mathrm{Rf}=0.20$ (hexane:ethyl acetate $1: 1 \mathrm{v} / \mathrm{v}) ;$ IR (ATR) $v_{\max } / \mathrm{cm}^{-1} 2922,1778,1717,1614,727$; ${ }^{1} \mathrm{H}$ NMR $\left(400 \mathrm{MHz}, \mathrm{CDCl}_{3}\right) \delta$ 7.84-7.88 (m, 2H), 7.71-7.75 (m, 2H), $7.34(\mathrm{dd}, J 7.4,0.8,1 \mathrm{H}), 7.28(\mathrm{td}, J 7.8,1.2,1 \mathrm{H})$, 7.19 (brd, $J 7.8,1 \mathrm{H}), 7.05(\mathrm{td}, J 7.4,1.2,1 \mathrm{H}), 5.71(\mathrm{~d}$, $J 14.1,1 \mathrm{H}), 5.56(\mathrm{~d}, J 14.1,1 \mathrm{H}), 3.18(\mathrm{~d}, J 16.8,1 \mathrm{H}), 2.96$ (d, $J 16.8,1 \mathrm{H}), 2.19(\mathrm{~s}, 3 \mathrm{H}) ;{ }^{13} \mathrm{C}$ NMR $\left(100 \mathrm{MHz}, \mathrm{CDCl}_{3}\right)$

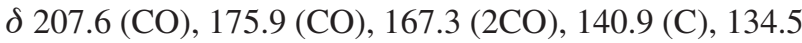
$(2 \mathrm{CH}), 131.6(2 \mathrm{C}), 130.2(\mathrm{CH}), 129.3(\mathrm{CH}), 124.0(\mathrm{CH})$, $123.8(2 \mathrm{CH}), 123.6(\mathrm{CH}), 109.7(\mathrm{CH}), 74.3(\mathrm{C}), 48.8$ $\left(\mathrm{CH}_{2}\right), 42.5\left(\mathrm{CH}_{2}\right), 31.5\left(\mathrm{CH}_{3}\right)$; HRESIMS $\mathrm{m} / z$, calcd. for $\mathrm{C}_{20} \mathrm{H}_{16} \mathrm{~N}_{2} \mathrm{NaO}_{5}{ }^{+}[\mathrm{M}+\mathrm{Na}]^{+}:$387.0951, found: 387.0936.

\section{Compound 2d (1-(4-fluoro-benzyl)-1H-indole-2,3-dione)}

It was obtained as orange powder in $55.13 \%$ yield $(0.048 \mathrm{~g}, 0.18 \mathrm{mmol})$. TLC: $\mathrm{Rf}=0.58$ (hexane:ethyl acetate $7: 3 \mathrm{v} / \mathrm{v}$ ); IR (ATR) $v_{\max } / \mathrm{cm}^{-1} 2924,1726,1608$, 762; ${ }^{1} \mathrm{H}$ NMR data were compared with the literature. ${ }^{36,37}$ HRESIMS $m / z$, calcd. for $\mathrm{C}_{15} \mathrm{H}_{11} \mathrm{FNO}_{2}{ }^{+}[\mathrm{M}+\mathrm{H}]^{+}: 256.0768$, found: 256.0764 ; $\mathrm{HRESIMS} \mathrm{m} / z$, calcd. for $\mathrm{C}_{15} \mathrm{H}_{10} \mathrm{FNNaO}_{2}{ }^{+}$ $[\mathrm{M}+\mathrm{Na}]^{+}: 278.0588$, found: 278.0583; HRESIMS $\mathrm{m} / \mathrm{z}$, calcd. for $\mathrm{C}_{30} \mathrm{H}_{20} \mathrm{~F}_{2} \mathrm{~N}_{2} \mathrm{NaO}_{4}{ }^{+}[2 \mathrm{M}+\mathrm{Na}]^{+}: 533.1283$, found: 533.1269.

Compound 2 e (1-(2-benzenesulfonylmethyl-benzyl)$1 \mathrm{H}$-indole-2,3-dione)

It was obtained as orange powder in quantitative yield. TLC: $\mathrm{Rf}=0.57$ (hexane:ethyl acetate 1:1 v/v); IR (ATR) $v_{\max } / \mathrm{cm}^{-1} 3497,1739,1663,1609,1141,721 ;{ }^{1} \mathrm{H}$ NMR $\left(400 \mathrm{MHz}\right.$, DMSO- $\left.d_{6}\right) \delta$ 7.85-7.89 (m, 2H), 7.78 (tt, $J$ 7.4, $1.2,1 \mathrm{H}), 7.66(\mathrm{td}, J 7.4,1.6,2 \mathrm{H}), 7.60(\mathrm{dd}, J 7.4,1.2,1 \mathrm{H})$, $7.56(\mathrm{td}, J 7.8,1.2,1 \mathrm{H}), 7.40(\mathrm{dd}, J 7.8,1.2,1 \mathrm{H}), 7.27(\mathrm{td}$, $J 7.4,1.2,1 \mathrm{H}), 7.22(\mathrm{td}, J 7.4,1.2,1 \mathrm{H}), 7.10-7.16(\mathrm{~m}, 2 \mathrm{H})$, $6.80\left(\mathrm{~d}, J\right.$ 8.2, 1H), 5.07 (s, 2H), $4.91(\mathrm{~s}, 2 \mathrm{H}) ;{ }^{13} \mathrm{C} \mathrm{NMR}$
(100 MHz, DMSO- $\left.d_{6}\right) \delta 183.0(\mathrm{CO}), 158.6(\mathrm{CO}), 150.2$ (C), $138.8(\mathrm{C}), 137.8(\mathrm{CH}), 135.3(\mathrm{C}), 134.1(\mathrm{CH}), 133.4$ $(\mathrm{CH}), 129.4(2 \mathrm{CH}), 129.0(\mathrm{CH}), 128.1(2 \mathrm{CH}), 127.3(\mathrm{CH})$, $127.1(\mathrm{CH}), 126.3(\mathrm{C}), 124.4(\mathrm{CH}), 123.4(\mathrm{CH}), 118.0(\mathrm{C})$, 111.2 $(\mathrm{CH}), 57.9\left(\mathrm{CH}_{2}\right), 41.1\left(\mathrm{CH}_{2}\right)$; HRESIMS $\mathrm{m} / z$, calcd. for $\mathrm{C}_{22} \mathrm{H}_{17} \mathrm{NNaO}_{4} \mathrm{~S}^{+}[\mathrm{M}+\mathrm{Na}]^{+}: 414.0770$, found: 414.0768; HRESIMS $\mathrm{m} / \mathrm{z}$, calcd. for $\mathrm{C}_{22} \mathrm{H}_{17} \mathrm{KNO}_{4} \mathrm{~S}^{+}[\mathrm{M}+\mathrm{K}]^{+}$: 430.0510, found: 430.0509; HRESIMS $\mathrm{m} / \mathrm{z}$, calcd. for $\mathrm{C}_{44} \mathrm{H}_{34} \mathrm{~N}_{2} \mathrm{NaO}_{8} \mathrm{~S}_{2}{ }^{+}[2 \mathrm{M}+\mathrm{Na}]^{+}:$805.1649, found: 805.1640.

\section{Compound $2 f$ (1-prop-2-ynyl-1 $H$-indole-2,3-dione)}

It was obtained as orange powder in quantitative yield. TLC: $\mathrm{Rf}=0.75$ (hexane:ethyl acetate $1: 1 \mathrm{v} / \mathrm{v}$ ); IR (ATR) $v_{\max } / \mathrm{cm}^{-1} 3263,2360,1735,1614,1343,677 ;{ }^{1} \mathrm{H}$ and ${ }^{13} \mathrm{C}$ NMR data were compared with the literature. ${ }^{38,39}$ HRESIMS $\mathrm{m} / z$, calcd. for $\mathrm{C}_{11} \mathrm{H}_{7} \mathrm{NNaO}_{2}{ }^{+}[\mathrm{M}+\mathrm{Na}]^{+}$: 208.0369, found: 208.0369; HRESIMS $\mathrm{m} / \mathrm{z}$, calcd. for $\mathrm{C}_{22} \mathrm{H}_{14} \mathrm{~N}_{2} \mathrm{NaO}_{4}^{+}[2 \mathrm{M}+\mathrm{Na}]^{+}:$393.0846, found: 393.0848.

Compound 4h (1-[1-(4-trifluoromethyl-phenyl)-1H-[1,2,3] triazol-4-ylmethyl]-1 $\mathrm{H}$-indole-2,3-dione)

It was obtained as orange powder in $70.38 \%$ yield $(0.141 \mathrm{~g}, 0.37 \mathrm{mmol})$. TLC: $\mathrm{Rf}=0.67$ (hexane:ethyl acetate $1: 1 \mathrm{v} / \mathrm{v})$; IR (ATR) $v_{\max } / \mathrm{cm}^{-1} 3145,1739,1721,1612,1154$, 751; ${ }^{1} \mathrm{H}$ NMR (400 MHz, DMSO- $d_{6}$ ) $\delta 8.98(\mathrm{~s}, 1 \mathrm{H}), 8.10$ (d, J 8.6, 2H), $7.96(\mathrm{~d}, J 8.6,2 \mathrm{H}), 7.64$ (brt, $J 7.8,1 \mathrm{H}), 7.59$ (brd, $J 7.4,1 \mathrm{H}$ ), 7.20 (brd, $J 8.2,1 \mathrm{H}), 7.15$ (brt, $J 7.8,1 \mathrm{H}$ ), 5.08 (s, 2H); ${ }^{13} \mathrm{C} \mathrm{NMR}\left(100 \mathrm{MHz}\right.$, DMSO- $\left.d_{6}\right) \delta 183.1(\mathrm{CO})$, 158.0 (CO), $150.1(\mathrm{C}), 143.3(\mathrm{C}), 139.3(\mathrm{C}), 138.2(\mathrm{CH})$, $127.3(\mathrm{C}), 127.3(2 \mathrm{CH}), 125.2(2 \mathrm{CH}), 124.6(\mathrm{CH}), 123.2$ $(\mathrm{CH}), 122.1(\mathrm{CH}), 120.6(\mathrm{C}), 117.8(\mathrm{C}), 111.2(\mathrm{C}), 35.0$ $\left(\mathrm{CH}_{2}\right)$; HRESIMS $m / z$, calcd. for $\mathrm{C}_{18} \mathrm{H}_{12} \mathrm{~F}_{3} \mathrm{~N}_{4} \mathrm{O}_{2}{ }^{+}[\mathrm{M}+\mathrm{H}]^{+}$: 373.0907, found: 373.0907; HRESIMS $\mathrm{m} / \mathrm{z}$, calcd. for $\mathrm{C}_{18} \mathrm{H}_{11} \mathrm{~F}_{3} \mathrm{~N}_{4} \mathrm{NaO}_{2}{ }^{+}[\mathrm{M}+\mathrm{Na}]^{+}:$395.0726, found: 395.0720; HRESIMS $\mathrm{m} / z$, calcd. for $\mathrm{C}_{18} \mathrm{H}_{11} \mathrm{~F}_{3} \mathrm{KN}_{4} \mathrm{O}_{2}{ }^{+}[\mathrm{M}+\mathrm{K}]^{+}$: 411.0466, found: 411.0486; HRESIMS $\mathrm{m} / \mathrm{z}$, calcd. for $\mathrm{C}_{36} \mathrm{H}_{22} \mathrm{~F}_{6} \mathrm{~N}_{8} \mathrm{NaO}_{4}{ }^{+}[2 \mathrm{M}+\mathrm{Na}]^{+}:$767.1560, found: 767.1529.

Compound 4i (1-[1-(2-methoxy-phenyl)-1H-[1,2,3]triazol4-ylmethyl]-1H-indole-2,3-dione)

It was obtained as orange powder in $16.66 \%$ yield $(0.032 \mathrm{~g}, 0.09 \mathrm{mmol})$. TLC: $\mathrm{Rf}=0.48$ (hexane:ethyl acetate $1: 1 \mathrm{v} / \mathrm{v}$ ); IR (ATR) $v_{\max } / \mathrm{cm}^{-1} 2925,1741,1613$, 1005,$752 ;{ }^{1} \mathrm{H}$ NMR (400 MHz, DMSO- $\left.d_{6}\right) \delta 8.51(\mathrm{~s}, 1 \mathrm{H})$, 7.65 (brt, J 7.8, 1H), 7.57 (brd, J 7.4, 2H), 7.51 (brt, $J$ 8.2, $1 \mathrm{H}), 7.29$ (brd, $J$ 8.2, 1H), 7.23 (d, J 7.8, 1H), 7.09-7.16 (m, 2H), 5.06 (s, 2H), 3.81 (s, 3H); ${ }^{13} \mathrm{C}$ NMR $(100 \mathrm{MHz}$, DMSO- $\left.d_{6}\right) \delta 183.1(\mathrm{CO}), 157.9(\mathrm{CO}), 151.6(\mathrm{C}), 150.2$ (C), $141.3(\mathrm{C}), 138.1(\mathrm{CH}), 130.8(\mathrm{C}), 125.7(2 \mathrm{CH})$, $125.6(\mathrm{CH}), 124.5(\mathrm{CH}), 123.4(\mathrm{CH}), 120.9(\mathrm{CH}), 117.7$ (C), $113.1(\mathrm{CH}), 111.3(\mathrm{CH}), 56.2\left(\mathrm{CH}_{3}\right), 35.0\left(\mathrm{CH}_{2}\right)$; 
HRESIMS $m / z$, calcd. for $\mathrm{C}_{18} \mathrm{H}_{15} \mathrm{~N}_{4} \mathrm{O}_{3}{ }^{+}[\mathrm{M}+\mathrm{H}]^{+}: 335.1139$, found: 335.1140; HRESIMS $m / z$, calcd. for $\mathrm{C}_{18} \mathrm{H}_{14} \mathrm{~N}_{4} \mathrm{NaO}_{3}{ }^{+}$ $[\mathrm{M}+\mathrm{Na}]^{+}:$357.0958, found: 357.0977 .

Compound 4j (1-[1-(4-bromo-phenyl)-1H-[1,2,3]triazol4-ylmethyl]-1H-indole-2,3-dione)

It was obtained as orange gel in $24.12 \%$ yield $(0.050 \mathrm{~g}$, $0.12 \mathrm{mmol}$ ). TLC: $\mathrm{Rf}=0.61$ (hexane:ethyl acetate $1: 1 \mathrm{v} / \mathrm{v}$ ); IR (ATR) $v_{\max } / \mathrm{cm}^{-1} 2917,1735,1718,1496,749 ;{ }^{1} \mathrm{H}$ NMR $\left(400 \mathrm{MHz}, \mathrm{DMSO}-d_{6}\right) \delta 8.88(\mathrm{~s}, 1 \mathrm{H}), 7.82(\mathrm{~d}, J 9.0,2 \mathrm{H})$, $7.78(\mathrm{~d}, J 9.0,2 \mathrm{H}), 7.64(\mathrm{td}, J 7.8,1.2,1 \mathrm{H}), 7.59$ (brd, $J 7.4,1 \mathrm{H}), 7.19$ (brd, J 7.8, 1H), $7.14(\mathrm{t}, J 7.4,1 \mathrm{H}), 5.06$ (s, $2 \mathrm{H}) ;{ }^{13} \mathrm{C}$ NMR (100 MHz, DMSO- $\left.d_{6}\right) \delta 183.1$ (CO), 158.0 (CO), $150.1(\mathrm{C}), 143.0(\mathrm{C}), 138.2(\mathrm{CH}), 135.7$ (C), 132.8 (C), $124.5(\mathrm{CH}), 123.5(\mathrm{CH}), 121.9(3 \mathrm{CH}), 121.4(2 \mathrm{CH})$, $117.7(\mathrm{C}), 111.1(\mathrm{CH}), 35.0\left(\mathrm{CH}_{2}\right)$; HRESIMS $\mathrm{m} / z$, calcd. for $\mathrm{C}_{17} \mathrm{H}_{12} \mathrm{BrN}_{4} \mathrm{O}_{2}{ }^{+}[\mathrm{M}+\mathrm{H}]^{+}:$383.0138, found: 383.0139 .

Compound 4k (1-[1-(2-benzenesulfonylmethyl-benzyl)$1 H$-[1,2,3]triazol-4-ylmethyl]- $1 H$-indole-2,3-dione)

It was obtained as orange powder in $19.43 \%$ yield (0.012 g, $0.02 \mathrm{mmol})$. TLC: $\mathrm{Rf}=0.51$ (hexane:ethyl acetate $1: 1 \mathrm{v} / \mathrm{v})$; IR (ATR) $v_{\max } / \mathrm{cm}^{-1} 2924,1734,1610,1262,755$; ${ }^{1} \mathrm{H}$ NMR (400 MHz, DMSO- $d_{6}$ ) $\delta 8.14(\mathrm{~s}, 1 \mathrm{H}), 7.80(\mathrm{~m}$, $2 \mathrm{H}), 7.75$ (dt, $J 8.2,1.2,1 \mathrm{H}), 7.66(\mathrm{~m}, 2 \mathrm{H}), 7.60$ (dd, J 7.8, $1.2,1 \mathrm{H}), 7.55(\mathrm{dd}, J 7.4,1.2,1 \mathrm{H}), 7.30(\mathrm{td}, J 7.4,1.5,1 \mathrm{H})$, $7.24(\mathrm{td}, J 7.4,1.5,1 \mathrm{H}), 7.08-7.14(\mathrm{~m}, 3 \mathrm{H}), 7.00$ (dd, J 7.8, $0.8,1 \mathrm{H}), 5.63(\mathrm{~s}, 2 \mathrm{H}), 4.95(\mathrm{~s}, 2 \mathrm{H}), 4.87(\mathrm{~s}, 2 \mathrm{H}) ;{ }^{13} \mathrm{C} \mathrm{NMR}$ $\left(100 \mathrm{MHz}, \mathrm{DMSO}-d_{6}\right) \delta 183.1(\mathrm{CO}), 157.9(\mathrm{CO}), 150.2$ (C), $142.0(\mathrm{C}), 138.5(\mathrm{C}), 138.1(\mathrm{CH}), 136.4(\mathrm{C}), 134.2$ $(\mathrm{CH}), 133.2(\mathrm{CH}), 129.4(2 \mathrm{CH}), 129.3(\mathrm{CH}), 128.7(\mathrm{CH})$, $128.2(\mathrm{CH}), 128.1(2 \mathrm{CH}), 126.6(\mathrm{C}), 124.5(\mathrm{CH}), 124.1$ $(\mathrm{CH}), 123.5(\mathrm{CH}), 117.7(\mathrm{C}), 111.2(\mathrm{CH}), 57.8\left(\mathrm{CH}_{2}\right), 50.2$ $\left(\mathrm{CH}_{2}\right), 35.1\left(\mathrm{CH}_{2}\right)$; HRESIMS $\mathrm{m} / z$, calcd. for $\mathrm{C}_{25} \mathrm{H}_{21} \mathrm{~N}_{4} \mathrm{O}_{4} \mathrm{~S}^{+}$ $[\mathrm{M}+\mathrm{H}]^{+}:$473.1278, found: 473.1275; HRESIMS $\mathrm{m} / \mathrm{z}$, calcd. for $\mathrm{C}_{25} \mathrm{H}_{20} \mathrm{~N}_{4} \mathrm{NaO}_{4} \mathrm{~S}^{+}[\mathrm{M}+\mathrm{Na}]^{+}: 495.1097$, found: 495.1092; HRESIMS $\mathrm{m} / \mathrm{z}$, calcd. for $\mathrm{C}_{25} \mathrm{H}_{20} \mathrm{KN}_{4} \mathrm{O}_{4} \mathrm{~S}^{+}$ $[\mathrm{M}+\mathrm{K}]^{+}$: 511.0837, found: 511.0843.

Compound 4I (4-[4-(2,3-dioxo-2,3-dihydro-indol1-ylmethyl)-[1,2,3]triazol-1-ylmethyl]-benzoic acid)

It was obtained as orange powder in $51.44 \%$ yield $(0.030 \mathrm{~g}, 0.08 \mathrm{mmol})$. TLC: $\mathrm{Rf}=0.27$ (hexane:ethyl acetate $1: 1 \mathrm{v} / \mathrm{v})$; IR (ATR) $v_{\max } / \mathrm{cm}^{-1} 3368,2925,1713,1610,1608$, 1380,$747 ;{ }^{1} \mathrm{H}$ NMR (400 MHz, DMSO- $\left.d_{6}\right) \delta 8.20(\mathrm{~s}, 1 \mathrm{H})$, $7.84(\mathrm{~d}, J 8.2,2 \mathrm{H}), 7.62(\mathrm{td}, J 7.8,1.2,1 \mathrm{H}), 7.55$ (brd, J 7.4, $1 \mathrm{H}), 7.16(\mathrm{~d}, J \mathrm{~d} .2,2 \mathrm{H}), 7.12(\mathrm{~m}, 2 \mathrm{H}), 5.55(\mathrm{~s}, 2 \mathrm{H}), 4.95$ $(\mathrm{s}, 2 \mathrm{H}) ;{ }^{13} \mathrm{C}$ NMR (100 MHz, DMSO- $\left.d_{6}\right) \delta 183.1(\mathrm{CO})$, $169.5(\mathrm{COOH}), 157.8(\mathrm{CO}), 150.2(\mathrm{C}), 141.7(\mathrm{C}), 139.5$ (C), $138.1(\mathrm{CH}), 136.5(\mathrm{C}), 129.4(2 \mathrm{CH}), 126.8(2 \mathrm{CH})$, $124.5(\mathrm{CH}), 123.8(\mathrm{CH}), 123.4(\mathrm{CH}), 117.6(\mathrm{C}), 111.2$
(CH), $52.8\left(\mathrm{CH}_{2}\right), 35.1\left(\mathrm{CH}_{2}\right)$. HRESIMS $\mathrm{m} / \mathrm{z}$, calcd. for $\mathrm{C}_{19} \mathrm{H}_{13} \mathrm{~N}_{4} \mathrm{O}_{4}^{-}[\mathrm{M}-\mathrm{H}]^{-}:$361.0942, found: 361.0941 .

\section{Molecular docking}

Docking assays were performed using the program Gold Suite 5.1 (Genetic Optimization for Ligand Docking 5.1) (CCDC Software Limited). ${ }^{40}$ A genetic algorithm of the crystallographic structure of GSK-3 $\beta$ from Homo sapiens (code protein data bank (PDB): 1Q3D), at $2.2 \AA$ resolution, was used. The binding mode of the compounds $\mathbf{2 b}$ and $\mathbf{2 e}$ was predicted. The predicted binding modes showed that both isatin derivatives exhibit similar complementarity (similar to staurosporine, with a score value 65.95), to the ATP-binding site of GSK-3 $\beta$ (PDB code 1Q3D). GOLD was used by a GoldScore fitness function, a molecular mechanism for the calculation of binding positions of ligand. Docking simulations were performed inside a $10 \AA$ radius sphere centered at the ligand in chain $\mathrm{A}$, and using the pattern parameters available, namely a population of 100 conformers, 100,000 operations, 95 mutations and 95 crossovers. The docked lowest-energy structure has a root mean square deviation (RMSD) of $0.75 \AA$ from the corresponding crystallographic complex.

\section{Biological assays}

The $N$-alkyl isatin derivatives (2a-e and $\mathbf{4 h}-\mathbf{l}$ ) were evaluated for their potential inhibitory effect against the GSK-3 $\beta$ enzyme. All compounds were diluted in dimethyl sulfoxide (DMSO). In all experiments, $0.2 \mu \mathrm{g} \mu \mathrm{L}^{-1}$ of GSK-3 $\beta$ substrate, $25 \mu \mathrm{M}$ of ATP and $1 \mathrm{ng}$ of GSK-3 $\beta$ enzyme were incubated together with the compounds under study for $60 \mathrm{~min}$. A commercial kit of luminescent kinase assay was used according to the supplier's instructions (Promega, V9371, Brazil). For the calculation of the half maximal inhibitory concentration $\left(\mathrm{IC}_{50}\right)$, a two-fold dilution was performed across each 96-well plate, starting with the highest isatin concentration $(25,50$ or $100 \mu \mathrm{M}$ depending on the isatin solubility and availability) up to $0.1 \mu \mathrm{M}$. For each test compound, a dose-response curve was generated. Results were represented as $\log$ (concentration of inhibitor) vs. response (Figure S71, Supplementary Information (SI) section), and, using the GraphPad program ${ }^{41}$ and a non-linear fit of the data, the $\mathrm{IC}_{50}$ value were determined.

\section{Results and Discussion}

In this work, comercial isatin was used as a starting material for bimolecular nucleophilic substitution and a click chemistry approach to develop all described chemical 
reactions. These reactions provide 11 semisynthetic isatin derivatives, seven of which are new. Despite their simple chemical structures, their biological activities were found to be notable. All 11 compounds were characterized using IR, NMR and HRMS.

The NMR data for all obtained compounds related to the isatin moiety were practically the same. We observed four signals related to aromatic hydrogens in the ${ }^{1} \mathrm{H}$ NMR spectra. The coupling constant of these hydrogens is characteristic of a 1,2-dissubstitute aromatic ring. The ${ }^{13} \mathrm{C}$ NMR spectra shows two signals at approximately $\delta 158$ and 182 ppm related to the carbons of the ketone and amide functions of isatin.

The HRESIMS of $\mathbf{2 a}$ were performed in a positive mode and showed the molecular ion peak at $\mathrm{m} / \mathrm{z} 368.0817$ in accordance with the molecular formula $\mathrm{C}_{23} \mathrm{H}_{13} \mathrm{NO}_{4}$. In the IR spectra, two stretching vibration bands of the $\mathrm{C}=\mathrm{O}$ bonds were observed at 1673 and $1605 \mathrm{~cm}^{-1}$, related to the carbonyl groups of the anthraquinone moiety. Aside from the characteristic isatin signals, the ${ }^{1} \mathrm{H}$ and ${ }^{13} \mathrm{C}$ NMR spectra of $2 \mathbf{a}$ showed signals at $\delta 5.10 \mathrm{ppm}(\mathrm{s}, 2 \mathrm{H})$ and $\delta 43.8 \mathrm{ppm}$, respectively, related to the methylene group attached to pyrrole-2,3-dione ring. This signal shows the coupling between isatin and the anthraquinone moiety. Additional aromatic signals were observed in the range $\delta 7.76$ to $8.31 \mathrm{ppm}$ and were attributed to the anthraquinone ring. In the ${ }^{13} \mathrm{C}$ NMR spectrum, two signals were observed at $\delta 182.5$ and $182.7 \mathrm{ppm}$ attributed to the carbonyl groups of the anthraquinone rings. Additional signals from the aromatic carbons of the anthraquinone ring were observed in the range of $\delta 127.3-141.3 \mathrm{ppm}$.

Compounds $\mathbf{2 b}$ and $\mathbf{2 d}$ are known, and the structures were confirmed by comparing the data obtained to results reported in the literature. ${ }^{33-37}$

For $\mathbf{2 e}$, the data obtained with HRESIMS established the molecular formula to be $\mathrm{C}_{22} \mathrm{H}_{17} \mathrm{NO}_{4} \mathrm{~S}$. The IR spectrum shows a characteristic absorption band at $1141 \mathrm{~cm}^{-1}$ related to the sulfone group stretch. In the ${ }^{1} \mathrm{H}$ NMR spectrum signals at $\delta 5.07 \mathrm{ppm}(\mathrm{s}, 2 \mathrm{H})$, related to the methylene group attached to pyrrole-2,3-dione ring, and $\delta 4.91 \mathrm{ppm}$ (s, $2 \mathrm{H})$, related to the other methylene group, were observed. Four aromatic hydrogens signals in the range $\delta$ 7.10-7.40 ppm were observed, which, together with the observed coupling constant, allowed us to determine the presence of a 1,2-dissubstitute aromatic ring in the structure. Additionally, signals in the range of $\delta$ 7.66-7.85 ppm associated with aromatic hydrogens of other aryl groups, along with the observed coupling constant, permitted us to determine the presence of a monosubstituted ring in the structure, confirming the final structure of 2e. Despite the isatin signals, signals in the
${ }^{13} \mathrm{C}$ NMR spectrum related to two additional aryl groups were observed in addition to signals from two methylene carbons at $\delta 41.1$ and $57.9 \mathrm{ppm}$.

The compound $\mathbf{2 f}$ was prepared as a starting material to produce the triazole derivatives by click chemistry reactions with organic azides. Compound $\mathbf{2} \mathbf{f}$ is known and its NMR data were compared with data reported in the literature. ${ }^{38,39}$

As for compounds $\mathbf{2 a - f}$, signals related to isatin were also observed for the compounds $\mathbf{4 h}-\mathbf{l}$ and will not be discussed.

The molecular formulae of compounds $\mathbf{4 h}$-l were obtained by HRESIMS. The ${ }^{1} \mathrm{H}$ NMR spectra of $\mathbf{4 h}-\mathbf{l}$ showed a hydrogen singlet in the range $\delta$ 8.14-8.98 ppm correlated to the hydrogen of the triazole ring. Signals related to the methylene group attached to pyrrole-2,3-dione ring were observed in the range $\delta 4.95-5.08 \mathrm{ppm}$. The ${ }^{13} \mathrm{C}$ spectra of these compounds showed signals in the range $\delta$ 122.1-125.7 ppm and 141.3-143.3 ppm related to the triazole ring carbons.

The IR spectrum of $\mathbf{4 h}$ shows an absorption band at $1154 \mathrm{~cm}^{-1}$ related to the $\mathrm{C}-\mathrm{F}$ stretch. The ${ }^{1} \mathrm{H}$ NMR spectrum of $\mathbf{4 h}$ showed two signals at $\delta 7.96 \mathrm{ppm}(\mathrm{d}, J 8.6,2 \mathrm{H})$ and $8.10 \mathrm{ppm}(\mathrm{d}, J 8.6,2 \mathrm{H})$ attributed to a 1,4-dissubstitute aromatic ring. The ${ }^{13} \mathrm{C}$ NMR spectrum showed a signal from the aryl group attached to the triazole ring in the range $\delta 125.2-139.3 \mathrm{ppm}$, and a signal at $\delta 120.6 \mathrm{ppm}$ attributed to a $\mathrm{CF}_{3}$ carbon.

The IR spectrum of $\mathbf{4 i}$ shows an absorption band at $1005 \mathrm{~cm}^{-1}$ related to the stretch of alkyl-aryl-ether. The ${ }^{1} \mathrm{H}$ NMR spectrum of $\mathbf{4 i}$ showed signals of aromatic hydrogens in the range $\delta 7.09-7.57 \mathrm{ppm}$. The coupling constant analysis of these hydrogens suggested the presence of a 1,2-dissubstitute aromatic ring in the structure. An additional signal was observed at $\delta 3.81 \mathrm{ppm}$ indicating the presence of a methoxy group. In the ${ }^{13} \mathrm{C}$ NMR spectrum, signals were observed at $\delta 151.6 \mathrm{ppm}$, associated with a phenolic group, at $\delta 56.2 \mathrm{ppm}$ related to a methoxy carbon, and in the range $\delta$ 120.9-130.8 ppm due to aryl carbons.

Compound $\mathbf{4 j}$ is known in the literature, ${ }^{42}$ however, its NMR data have not been discussed in the previously published work. The IR spectrum of $\mathbf{4} \mathbf{j}$ shows a characteristic absorption band at $1496 \mathrm{~cm}^{-1}$ related to the $\mathrm{C}-\mathrm{Br}$ stretch. The ${ }^{1} \mathrm{H}$ NMR spectrum of $\mathbf{4 j}$ showed two signals at $\delta 7.78 \mathrm{ppm}(\mathrm{d}, J 9.0,2 \mathrm{H})$ and $7.82 \mathrm{ppm}(\mathrm{d}, J 9.0,2 \mathrm{H})$ attributed to a 1,4-dissubstitute aromatic ring. The ${ }^{13} \mathrm{C} N \mathrm{NM}$ spectrum showed signal of the aryl group attached to the triazole ring in the range $\delta 121.4-135.7 \mathrm{ppm}$ related to a bromobenzene ring.

For $4 \mathbf{k}$, the IR spectrum showed a characteristic absorption band at $1262 \mathrm{~cm}^{-1}$ related to the stretch of a sulfone group. In the ${ }^{1} \mathrm{H}$ NMR spectrum, an additional 
methylene group signal at $\delta 4.87 \mathrm{ppm}$ (s, 2H) was observed and was attributed to a methylene sulfone group. Four aromatic hydrogens signals in the range $\delta 7.00-7.30 \mathrm{ppm}$ were observed, which, together with the coupling constant, allowed us to determine the presence of a 1,2-dissubstitute aromatic ring in the structure. Additionally, signals from aromatic hydrogens of other aryl groups in the range $\delta$ 7.66-7.80 ppm were observed; coupling constant analysis allowed us to determine the presence a monosubstituted ring in the structure. Despite of the isatin signals, signals in the ${ }^{13} \mathrm{C}$ NMR spectrum related to two additional aryl groups in the range $\delta 126.6-138.5 \mathrm{ppm}$ were observed in addition to two methylene carbons at $\delta 50.2$ and $57.8 \mathrm{ppm}$.

The IR spectrum of $\mathbf{4} \mathbf{l}$ shows characteristic absorption bands at 3368,1713 and $1380 \mathrm{~cm}^{-1}$ related to the stretch of $\mathrm{O}-\mathrm{H}, \mathrm{C}=\mathrm{O}$ and $\mathrm{C}-\mathrm{O}$, respectively. The ${ }^{1} \mathrm{H}$ NMR spectrum of $4 \mathbf{l}$ showed two signals at $\delta 7.16 \mathrm{ppm}(\mathrm{d}, J 8.2,2 \mathrm{H})$ and $7.84 \mathrm{ppm}(\mathrm{d}, J 8.2,2 \mathrm{H})$ attributed to a 1,4-dissubstitute aromatic ring. An additional methylene group at $\delta 5.58 \mathrm{ppm}$ (s, 2H) was observed. The ${ }^{13} \mathrm{C}$ NMR spectrum showed a signal of the aryl group in the range $\delta 126.8-139.5 \mathrm{ppm}$, and a signal at $\delta 169.5 \mathrm{ppm}$ confirms the presence of a $\mathrm{COOH}$ group.

During the preparation of the $N$-alkyl isatin derivatives, interesting and unexpected events occurred regarding $\mathbf{2 c}$. Synthesis and biological properties of $\mathrm{N}$-alkyl isatin derivatives are broadly described in the literature..$^{16,43,44}$ These methods are simple and easy, and they differ from each other in the bases and solvents used. However, in our study, in addition to using bimolecular nucleophilic substitution to synthesize a convenient isatin derivative, we used potassium carbonate and $\mathrm{N}$-(bromomethyl) phthalimide to prepare a novel isatin derivative named $\mathbf{2 c}$ that resulted from a nucleophilic addition in the carbonyl group at position 3 of isatin. The proposed mechanism towards the synthesis of $\mathbf{2 c}$ is illustrated in Figure 1. Synthesis occurs via the enolate anion that is obtained from the reaction between potassium carbonate and acetone, which participates as both solvent and reagent. Thus, the nucleophile enolate anion attacks the carbonyl group at C-3 and consequently leads to the production of alcoxide anion and, finally, alcohol in 2c.

The HRESIMS of $\mathbf{2 c}$ was performed in the positive mode. The ion peak at $m / z 387.0936$ is consistent with the molecular formula $\mathrm{C}_{20} \mathrm{H}_{16} \mathrm{~N}_{2} \mathrm{O}_{5}$. The two signals observed in the ${ }^{1} \mathrm{H}$ NMR spectrum at $\delta 7.71-7.75 \mathrm{ppm}(\mathrm{m}, 2 \mathrm{H})$ and 7.84-7.88 ppm ( $\mathrm{m}, 2 \mathrm{H})$, and from the coupling constant we determined the presence of a 1,2-dissusbstitute symmetric aromatic ring. Th signal observed in the ${ }^{13} \mathrm{C}$ NMR spectrum at $\delta 167.3 \mathrm{ppm}$ confirmed the presence of the amide group in the phthalimide moiety. Two hydrogens at $\delta 5.71 \mathrm{ppm}$ $(\mathrm{d}, J 14.1,1 \mathrm{H})$ and $5.56 \mathrm{ppm}(\mathrm{d}, J 14.1,1 \mathrm{H})$ confirmed the presence of a methylene group.

Nucleophilic addition to the carbonyl group at position 3 was confirmed by the signal at $\delta 73.3 \mathrm{ppm}$ that demonstrated the reduction of the ketone group. Two hydrogens at $2.96 \mathrm{ppm}(\mathrm{d}, J 16.8,1 \mathrm{H})$ and $3.18 \mathrm{ppm}(\mathrm{d}, J 16.8,1 \mathrm{H})$ confirm the presence of other methylene groups in the structure. To finish, the carbon signals at $\delta 207.6$ and $31.5 \mathrm{ppm}$ show the nucleophilic addition of the enolate anion.

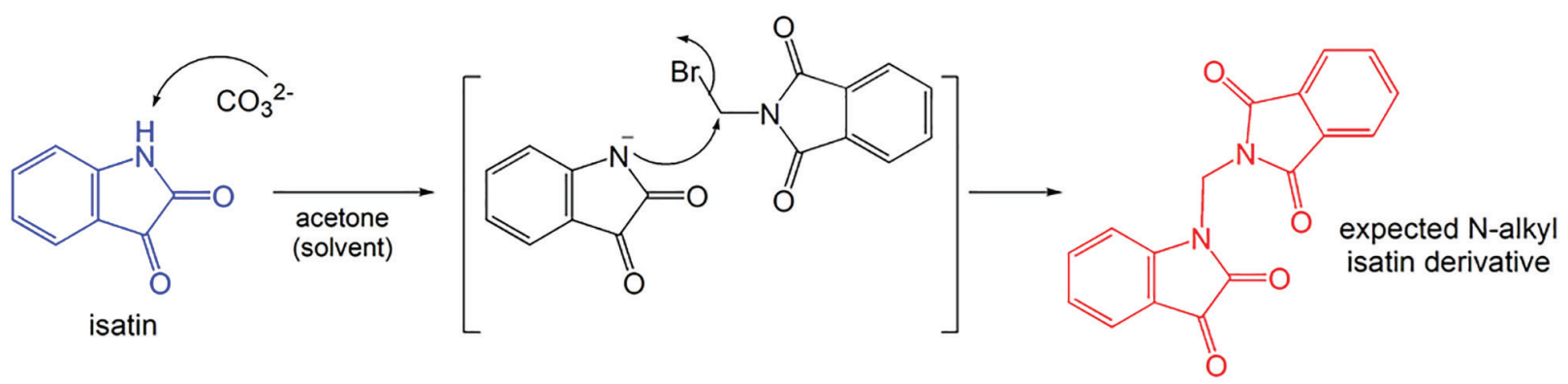

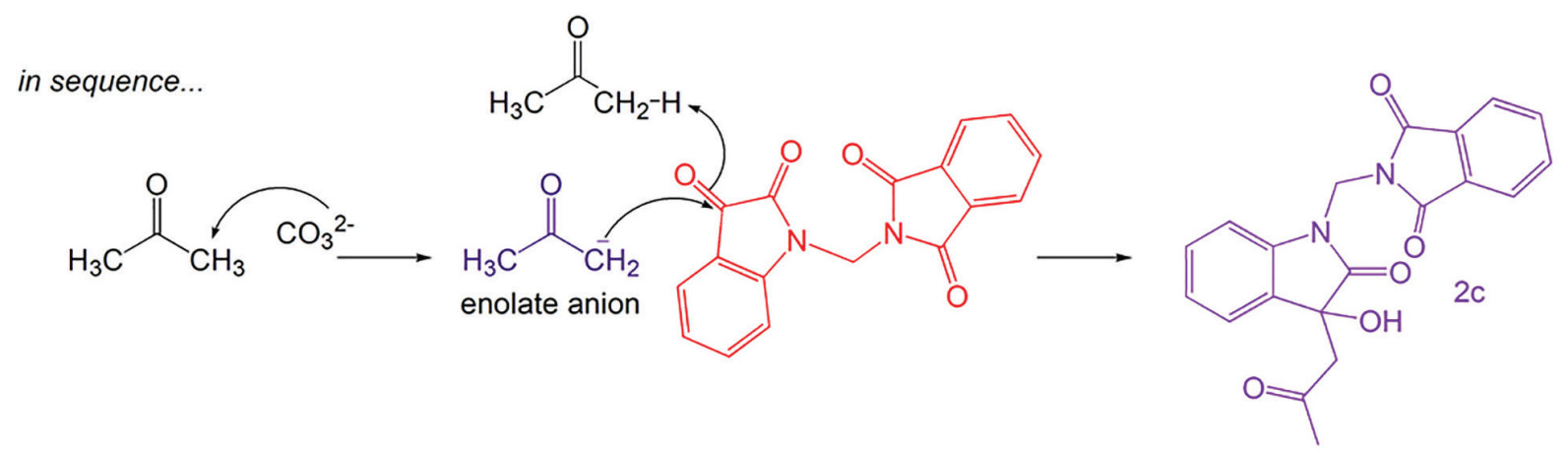

Figure 1. Proposed mechanism towards the synthesis of $\mathbf{2 c}$. 


\section{Docking studies on isatin derivatives}

Staurosporine is an alkaloid containing an indolo[2,3-a]carbazolole nucleus which has remarkable inhibitory activity against GSK-3 $\beta$ protein kinase, with an $\mathrm{IC}_{50}$ of $15 \mathrm{nM}$. This alkaloid was first obtained from Streptomyces staurosporeus, and its crystallographic structure in a complex with GSK-3 $\beta$ (PDB code 1Q3D) highlights important interactions related to enzymatic inhibition. Regarding the ATP-binding site in GSK-3 $\beta$, direct hydrogen bond interactions are disclosed involving N1 of staurosporine with the Asp133 carbonyl oxygen, and the $\mathrm{O} 5$ of staurosporine with the backbone nitrogen of Val135. A water-mediated hydrogen bond is also observed between the carbonyl group in Gln185 and N at position 4 in the glycosidic portion. ${ }^{45}$ Furthermore, a hydrogen-bonding network can be seen, consisting of the Thr138 residue, 4 water molecules as intermediates and finally the carbonyl group in Val135 (Figure 2). The fused carbazole portion in this alkaloid, which interacts with water molecules to form a cavity, has interesting hydrophobic interactions at the GSK-3 $\beta$ ATP-binding site. Ile62, Gly63, Gly65, Val70, Ala83, Asp133, Tyr134, Gln185, Asn186, Leu188, Cys199 and Asp200 are residues involved in hydrophobic interactions and are part of the buried surface area in the staurosporine-GSK-3 $\beta$ crystallographic structure. ${ }^{46}$

To gain a better understanding of the inhibitory activity of $\mathbf{2 b}$, we analysed its interactions with GSK-3 $\beta$. All docking runs applied the genetic algorithm of GOLD 5.1. The binding mode of compound $\mathbf{2 b}$ (the most active compound assayed) onto the GSK-3 $\beta$ ATP-binding site is depicted in Figure 3. All amino acid residues that had interactions with this isatin derivative are exhibited. In the binding mode, 2b (score value 43.33) is nicely complexed with GSK-3 $\beta$ and stabilized by two remarkable hydrogen bonds with important aminoacids at the catalytic site. Furthermore, a superposition of $\mathbf{2} \mathbf{b}$ and staurosporine reveals that both compounds are located at the edge of the ATP binding-site, suggesting that $\mathbf{2 b}$ acts as an ATP-mimetic inhibitor. The oxygen atom of the nitro group in $\mathbf{2} \mathbf{b}$ formed a hydrogen bond with the $-\mathrm{NH}_{2}$ moiety in the Asp200 residue (3.3 $\AA$ ), similar to the inhibitor indirubin-3'-monoxime. ${ }^{46}$ Another notable hydrogen bond is formed by the oxygen atom of the carbonyl group of $\mathbf{2} \mathbf{b}$ and the $-\mathrm{NH}_{2}$ group present in the side chain of Gly65 (3.0 ̊). This interaction is important since this residue contributes to the stabilization and surface area in the GSK-3 $\beta$ active site.
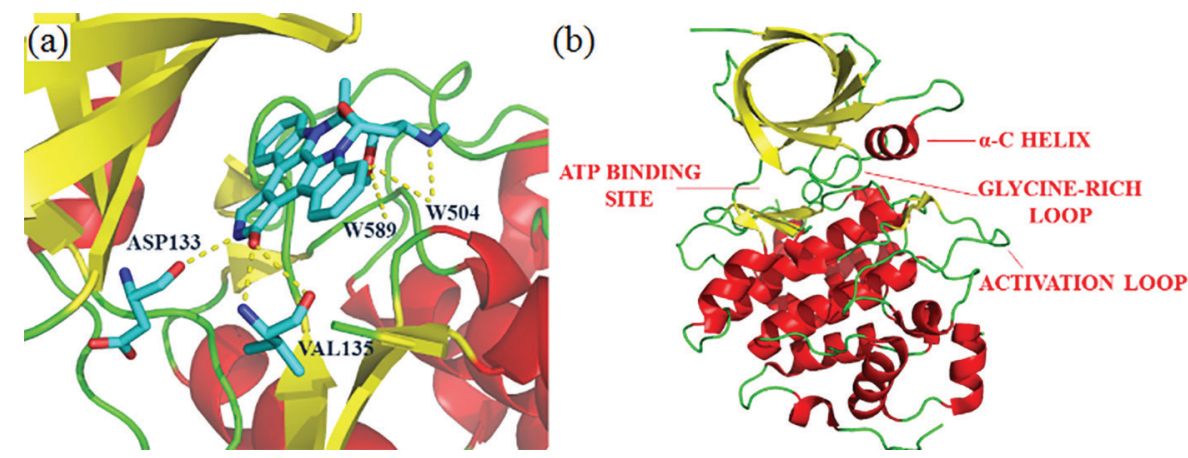

Figure 2. (a) Binding of staurosporine (blue) to GSK-3 $\beta$ with ASP133 and VAL135 aminoacids directly related to an inhibitory role; (b) crystallographic information of GSK-3 $\beta$ and its relevant groups.

(a)

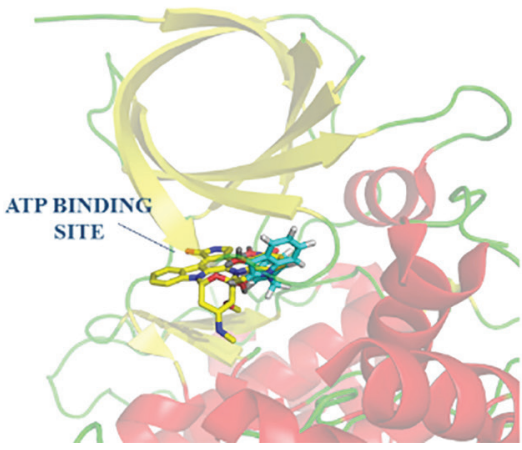

(b)

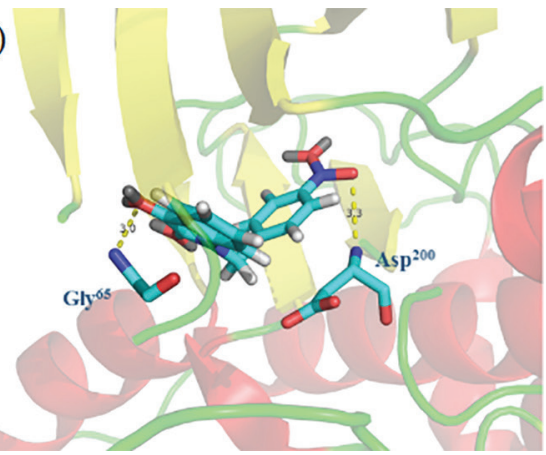

Figure 3. (a) Overview of the 3D structure of GSK-3 $\beta$ highlighting the region for catalysis or inhibitor binding. The structure of $\mathbf{2 b}$ (light blue, carbon) is close to the staurosporine structure (yellow, carbon) showing complementarity with the ATP binding site; (b) detailed view of $\mathbf{2 b}$ and nearby residues in the active site, with dashed lines showing hydrogen bonds and distances. All interactions shown are less than $4.5 \AA$ and therefore are capable of hydrogen bonding. 


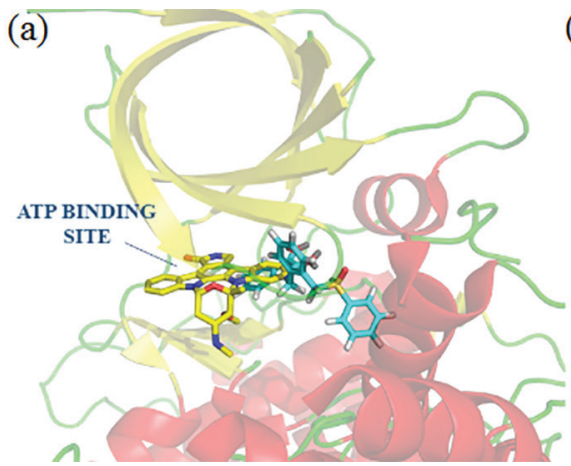

(b)

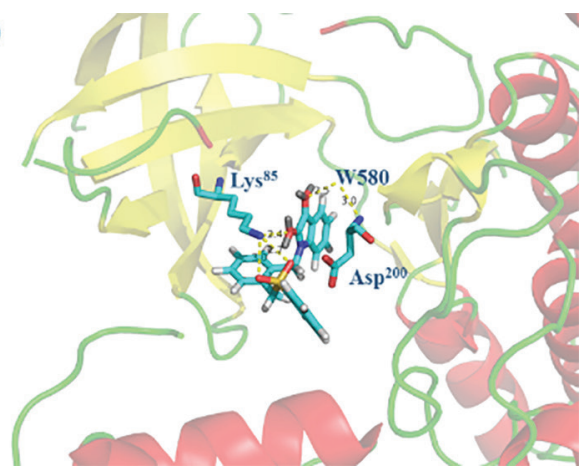

Figure 4. (a) Overview of the 3D structure of GSK-3ß. The structure of $2 \mathbf{e}$ (light blue, carbon) is close to the staurosporine structure (yellow, carbon) showing complementarity to the ATP-binding site; (b) detailed view of $\mathbf{2} \mathbf{e}$ and nearby residues in the active site. Dashed lines showing hydrogen bonds and distances.

With respect to the binding mode of the isatin derivative $\mathbf{2 e}$, which presented the best score value of 66.34, interactions with staurosporine highlight that $\mathbf{2 e}$ is capable of forming hydrogens bonds with the GSK-3 $\beta$ ATP binding site (Figure 4). In the binding mode prediction, complementarity of $2 \mathbf{e}$ to the ATP binding site on GSK-3 $\beta$ is observed, like $\mathbf{2 b}$ (as discussed above). Stabilization is achieved by two strong hydrogen bonds, between the amino terminal moiety in Lys85 and $(i)$ the oxygen atom in carbonyl at position 2 in isatin $(2.4 \AA)$, and (ii) the sulfonyl group (2.7, $3.0 \AA$ ). Moreover, a water-mediated hydrogen bond, W580, permits an interaction between carbonyl, at position 2 in this derivative, and the $-\mathrm{NH}_{2}$ moiety of the Asp200 residue.

The docking results confirmed that the aforementioned hydrogen bonding interactions might contribute to the powerful biological activities found for $\mathbf{2 b}$ and $\mathbf{2 e} N$-alkyl isatin derivatives. Moreover, these results contribute to a better understanding of important pharmacophoric features required to further develop isatin derivatives holding antiGSK-3 $\beta$ activity, namely contain voluminous groups, as seen in $\mathbf{2 e}$ structure, and/or electronegative groups, as seen in $\mathbf{2 b}$ structure. Table 1 summarizes the score value and interactions for chemical groups found to be interesting for inhibitory activity.

\section{Inhibitory effect of isatin derivatives on GSK-3ß enzyme}

The isatin derivative's ability to inhibit the GSK-3 $\beta$ enzyme was evaluated in vitro. The concentrations of inhibitor required to reach $50 \%$ enzymatic inhibition under the given experimental conditions $\left(\mathrm{IC}_{50}\right)$ are summarized in Table 2. As previously mentioned, staurosporine is described in the literature as a pattern inhibitor of GSK-3 $\beta$ with an $\mathrm{IC}_{50}$ value of $15 \mathrm{nM} .{ }^{47} \mathrm{~A}$ wide range of $\mathrm{IC}_{50}$ values were obtained for the different isatin derivatives tested and are related to the substituents at $N-1$ in the isatin building block.
Table 1. Binding mode prediction of isatin derivatives as GSK-3 $\beta$ inhibitors

\begin{tabular}{|c|c|c|}
\hline Compound & $\begin{array}{l}\text { Score } \\
\text { value }\end{array}$ & $\begin{array}{l}\text { Key amino acids to hydrogen bond interactions } \\
\text { in ATP-binding site }\end{array}$ \\
\hline $2 \mathbf{a}$ & 58.06 & $\begin{array}{c}-\mathrm{NH}_{2} \text { Lys85: } \mathrm{C}=\mathrm{O}(\mathrm{C} 2) 2.9 \AA \\
-\mathrm{NH}_{2} \text { Lys85: } \mathrm{C}=\mathrm{O} \text { (anthraquinone) } 2.8 \AA\end{array}$ \\
\hline $2 \mathrm{c}$ & 52.07 & $\begin{array}{c}-\mathrm{NH}_{2} \text { Lys85: C=O (phthalimide) } 2.6 \AA \\
-\mathrm{NH}_{2} \text { Lys } 183:-\mathrm{OH} 3.2 \AA\end{array}$ \\
\hline 2d & 42.67 & $-\mathrm{NH}_{2}$ Lys85: $\mathrm{C}=\mathrm{O}(\mathrm{C} 2) 2.5 \AA$ \\
\hline $4 h$ & 50.88 & $-\mathrm{NH}_{2}$ Lys85: $\mathrm{C}=\mathrm{O}(\mathrm{C} 2) 2.5 \AA$ \\
\hline $4 \mathbf{i}$ & 55.02 & -NH Gly65: C=O (C3) $3.1 \AA$ \\
\hline $4 j$ & 52.59 & $-\mathrm{NH}_{2}$ Lys85: $\mathrm{C}=\mathrm{O}(\mathrm{C} 2) 2.4 \AA$ \\
\hline $4 k$ & 65.43 & $\begin{array}{l}\text {-OH Ser66: C=O (C3) } 3.4 \AA \\
\text {-NH Phe67: } \mathrm{C}=\mathrm{O}(\mathrm{C} 2) 3.5 \AA\end{array}$ \\
\hline 41 & 58.83 & $\begin{array}{c}-\mathrm{NH} \text { Asp200: } \mathrm{C}=\mathrm{O}(\mathrm{COOH}) 3.4 \AA \\
-\mathrm{NH}_{2} \text { Lys85: } \mathrm{C}=\mathrm{O}(\mathrm{C} 3) 2.5 \AA\end{array}$ \\
\hline
\end{tabular}

Table 2. Inhibition effect of isatin derivatives against the GSK-3 $\beta$ enzyme

\begin{tabular}{lc}
\hline Compound & $\mathrm{IC}_{50}$ value $/ \mu \mathrm{M}$ \\
\hline $\mathbf{2 a}$ & 1.363 \\
$\mathbf{2 b}$ & $4.612 \times 10^{-1}$ \\
$\mathbf{2 c}$ & $1.531 \times 10^{2}$ \\
$\mathbf{2 d}$ & $4.106 \times 10^{1}$ \\
$\mathbf{2 e}$ & 5.397 \\
$\mathbf{4 h}$ & $7.320 \times 10^{-1}$ \\
$\mathbf{4 i}$ & $1.926 \times 10^{1}$ \\
$\mathbf{4 j}$ & 4.921 \\
$\mathbf{4 k}$ & 3.442 \\
$\mathbf{4 l}$ & 3.433 \\
\hline
\end{tabular}

$\mathrm{IC}_{50}$ : half maximal inhibitory concentration.

A strong inhibitory activity against the GSK-3ß enzyme was identified for all isatin derivatives studied, with $\mathbf{2 b}$ and $4 \mathrm{~h}$ derivatives being the most effective. Stronger inhibitory ability was correlated with the addition of bulkier and/or electronegative substituents. Comparing 
the isatin derivatives $\mathbf{2} \mathbf{b}$ and $\mathbf{2 d}$, both contain a parasubstituted benzene ring. However, $\mathbf{2} \mathbf{b}$, which has a nitro group as a substituent with greater electron-withdrawing effect, exhibited significantly better inhibitory ability $\left(\mathrm{IC}_{50}=4.612 \times 10^{-1} \mu \mathrm{M}\right)$ than $\mathbf{2 d}$, which has a fluorine group. Thus, the presence of more electronegative groups as substituents appears to be important. Compared with 2a and $\mathbf{2 e}$ which contain a less bulky groups, $\mathbf{2} \mathbf{b}$ has superior inhibitory ability. Regarding the triazolic isatin derivatives shown in Scheme 3, a similar correlation was given to the substituents of the benzyl ring. The isatin derivatives $\mathbf{4 h}, \mathbf{4 j}$ and $\mathbf{4 l}$ showed $\mathrm{IC}_{50}$ values significantly better than $4 \mathbf{i}\left(\mathrm{IC}_{50}=1.926 \times 10^{1} \mu \mathrm{M}\right)$, which has an electron-donor effect related to the $-\mathrm{OCH}_{3}$ group. Furthermore, of these para-substituted compounds, $4 \mathrm{~h}$ has a better $\mathrm{IC}_{50}$ value $(0.7323 \mu \mathrm{M})$, probably owing to the greater electronwithdrawing ability of the $-\mathrm{CF}_{3}$ group. Both $\mathbf{2 e}$ and $\mathbf{4 k}$ derivatives possess a 2-benzenesulfonylmethyl-benzyl as a substituent; however, it was found that the $\mathbf{4 k}$, spaced by a triazol ring, has an $\mathrm{IC}_{50}$ value, $3.442 \mu \mathrm{M}$, performing better than to $2 \mathrm{e}$, with an $\mathrm{IC}_{50}$ of $5.397 \mu \mathrm{M}$. These results are in accordance with the observation that bulkier groups are associated with greater inhibitory potential, further suggesting that the triazolic ring plays an important role. Finally, from analysis of the results for the $\mathbf{2 c}$ derivative, preserving the carbonyl portions of the isatin scaffold was found to be crucial; this derivative had the worst $\mathrm{IC}_{50}$ value, most likely because of the nucleophilic addition that occurs during its preparation.

\section{Conclusions}

Of all tested compounds, the $N$-alkyl isatin $\mathbf{2 b}$ and $\mathbf{4 h}$ derivatives, with $\mathrm{IC}_{50}$ values of 0.4612 and $0.7323 \mu \mathrm{M}$, respectively, were found to be the most effective against GSK-3 $\beta$ enzyme. This better activity was attributed to the presence of a group with greater electron-withdrawing ability in a para-position. Moreover, the existence of bulkier substituents was also found to be crucial to improve the inhibitory activity against GSK-3 $\beta$, as seen for $\mathbf{2 a}, \mathbf{2} \mathbf{e}$ and $\mathbf{4 k}$. Moreover, the binding mode prediction of $\mathbf{2} \mathbf{b}$ and 2e through docking studies provided better insight into the hydrogen bond interactions with the GSK-3 $\beta$ ATPbinding site.

\section{Supplementary Information}

Supplementary information (IR, NMR and HRMS) is available free of charge at http://jbcs.sbq.org.br as PDF file.

\section{Acknowledgments}

This study was funded by the Coordenação de Aperfeiçoamento de Pessoal de Nível Superior, Brasil (CAPES), Finance Code 001, by the National Council of Scientific and Technological Development (CNPq) and Foundation of Support to Research and Innovation of Espírito Santo (FAPES PPE-Agro No. 76418880/16). We also would like to acknowledge INCTBioNat (CNPq 465637/2014-0) for additional support and NCQP-UFES, as well as the Portuguese Foundation for Science and Technology (FCT) under the scope of the strategic funding of UID/BIO/04469/2019 unit and BioTecNorte operation (NORTE-01-0145-FEDER-000004) funded by the European Regional Development Fund under the scope of Norte2020, Programa Operacional Regional do Norte. L. R. R. also acknowledges her sabbatical leave fellowship (SFRH/BSAB/142991/2018) funded by FCT. D. F. is recipient of a doctoral fellowship (call NORTE-69-2015-15) funded by the European Social Fund under the scope of Norte2020, Programa Operacional Regional do Norte.

\section{References}

1. Erdmann, O. L.; J. Prakt. Chem. 1840, 19, 321.

2. Laurent, A.; Ann. Chim. Phys. 1840, 3, 393.

3. Khan, A. F.; Maalik, A.; Noor, T.; Zaidi, A.; Farooq, U.; Bukhari, S. M.; Trop. J. Pharm. Res. 2015, 14, 1937.

4. Gang, C.; Hui-Jun, S.; Zhang; M.; Fang, H.; Zhang, J.; Xiaojiang, H.; Jing-Rui, Z.; Chem. Cent. J. 2012, 6, 90.

5. Bergman, J.; Lindström, J. O.; Tilstam, U.; Tetrahedron 1985, 41, 2879.

6. Yoshikawa, M.; Murakami, T.; Kishi, A.; Sakurama, T.; Matsuda, H.; Nomura, M.; Matsuda, H.; Kubo, M.; Chem. Pharm. Bull. 1998, 46, 886.

7. Sandmeyer, T.; Helv. Chim. Acta 1919, 2, 234.

8. Silva, B. N. M.; Silva, B. V.; Silva, F. C.; Gonzaga, D. T. G.; Ferreira, V. F.; Pinto, A. C.; J. Braz. Chem. Soc. 2013, 24, 179.

9. Chaudhary, D. K.; Ahmad, S.; Maitya, S.; Alama, M. S.; Pharm. Lett. 2013, 5, 285.

10. Gil-Turnes, M. S.; Hay, M. E.; Fenical, W.; Science 1989, 246, 116.

11. Nandakumar, A.; Thirumurugan, P.; Perumal, P. T.; Vembu, P.; Ponnuswamy, M. N.; Ramesh, P.; Bioorg. Med. Chem. Lett. 2010, 14, 4252.

12. Panneerselvam, P.; Reddy, R. S.; Murali, K.; Kumar, N. R.; Pharma Chem. 2010, 2, 37.

13. Chaluvaraju, K. C.; Zaranappa, X.; Res. J. Pharm., Biol. Chem. Sci. 2011, 2, 541. 
14. Kang, I. J.; Wang, L. W.; Hsu, T. A.; Yueh, A.; Lee, C. C.; Lee, Y. C.; Lee, C. Y.; Chao, Y. S.; Shih, S. R.; Chern, J. H.; Bioorg. Med. Chem. Lett. 2011, 21, 1948.

15. Kandasamy, R.; Park, S. J.; Boyapalle, S.; Mohapatra, S.; Hellermann, G. R.; Lockey, R. F.; Moahapatra, S. S.; Int. Immunopharmacol. 2010, 10, 218.

16. Chen, G.; Wang, Y.; Hao, X.; Mu, S.; Sun, Q.; Chem. Cent. J. 2011, 5, 37.

17. Tan, H. Z.; Wang, W. M.; Shang, J. L.; Song, H. B.; Li, Z. M.; Wang, J. G.; Chin. J. Struct. Chem. 2011, 30, 502.

18. Wang, J.; Tan, H.; Li, Y.; Ma, Y.; Li, Z.; Guddat, L. W.; J. Agric. Food Chem. 2011, 59, 9892.

19. Krishnegowda, G.; Gowda, A. S. P.; Tagaram, H. R. S.; Carroll, K. F. S.-O'.; Irby, R. B.; Sharma, A. K.; Amin, S.; Bioorg. Med. Chem. 2011, 19, 6006.

20. Faivre, S.; Demetri, G.; Sargente, W.; Raymond, E.; Nat. Rev. Drug Discovery 2007, 6, 734.

21. Vinik, A. I.; Raymond, E.; Ther. Adv. Gastroenterol. 2013, 6, 396.

22. Singh, P.; Sharma, P.; Anand, A.; Bedi, P. M. S.; Kaur, T.; Saxena, A. K.; Kumar, V.; Eur. J. Med. Chem. 2012, 55, 455.

23. Damiens, E.; Baratte, B.; Marie, D.; Eisenbrand, G.; Meijer, L.; Oncogene 2001, 20, 3786.

24. Yu, J.; Zheng, J.; Lin, J.; Jin, L.; Yu, R.; Mak, S.; Hu, S.; Sun, H.; Wu, X.; Zhang, Z.; Lee, M.; Tsim, W.; Cell. Mol. Neurobiol. 2017, 37, 655 .

25. Embi, N.; Rylatt, D. B.; Cohen, P.; Eur. J. Biochem. 1980, 107, 519.

26. Woodgett, J. R.; EMBO J. 1990, 9, 2431.

27. ter Haar, E. In Glycogen Synthase Kinase 3 (GSK-3) and Its Inhibitors: Drug Discovery and Development; Martinez, A.; Castro, A.; Medina, M., eds.; John Wiley \& Sons: New Jersey, USA, 2006, ch. 4, p. 61.

28. Cross, D. A.; Alessi, D. R.; Cohen, P.; Anadjelkovich, M.; Hemmings, B. A.; Nature 1995, 378, 785.

29. ter Haar, E.; Coll, J. T.; Austen, D. A.; Hsiao, H. M.; Swenson, L.; Jain, J.; Nat. Struct. Mol. Biol. 2001, 8, 593.

30. Hughes, K.; Nokolakaki, E.; Plyte, S. E.; Totty, N. F.; Woodgett, J. R.; EMBO J. 1993, 12, 803.

31. Bax, B.; Carter, P. S.; Lewis, C.; Guy, A. R.; Bridges, A.; Tanner,
R.; Structure 2001, 9, 1143.

32. Dajani, R.; Fraser, E.; Roe, S. M.; Young, N.; Good, V.; Dale, T. C.; Pearl, L. H.; Cell 2001, 105, 721.

33. Furdas, S. D.; Shekfeh, S.; Kannan, S.; Sippl, W.; Jung, M.; Med. Chem. Commun. 2012, 3, 305.

34. Makhija, M. T.; Kasliwal, R. T.; Kulkarni, V. T.; Neamati, N.; Bioorg. Med. Chem. 2004, 12, 2317.

35. Gui, J.; Chen, G.; Cao, P.; Liao, J.; Tetrahedron: Asymmetry 2012, 23, 554.

36. Shi, F.; Tao, Z.-L.; Luo, S.-W.; Tu, S.-J.; Gong, L.-Z.; Chem. Eur. J. 2012, 18, 6885.

37. Tehrani, K. H. M. E.; Hashemi, M.; Hassan, M.; Kobarfard, F.; Mohebbi, S.; Chin. Chem. Lett. 2016, 27, 221.

38. Bouhfid, R.; Joly, N.; Essassi, E. M.; Lequart, V.; Massoui, M.; Martin, P.; Synth. Commun. 2011, 41, 2096.

39. Macpherson, L. J.; Dubin, A. E.; Evans, M. J.; Marr, F.; Schultz, P. G.; Cravatt, B. F.; Patapoutian, A.; Nature 2007, 445, 541.

40. Jones, G.; Willett, P.; Glen, R. C.; Leach, A. R.; Taylor, R.; J. Mol. Biol. 1997, 267, 727.

41. GraphPad Prism, 6.00 version; GraphPad Software Inc., San Diego, USA, 2018.

42. Jain, R.; Gahlyan, P.; Dwivedi, S.; Konwar, R.; Kumar, S.; Bhandari, M.; Arora, R.; Kakkar, R.; Kumar, R.; Prasad, A. K.; ChemistrySelect 2018, 3, 5263.

43. Pakravan, P.; Kashanian, S.; Khodaei, M. M.; Harding, F. J.; Pharmacol. Rep. 2013, 65, 313.

44. Shmidt, M. S.; Reverdito, A. M.; Kremenchuzky, L.; Perillo, I. A.; Blanco, M. M.; Molecules 2008, 13, 831.

45. Lamers, M. B. A. C.; Antson, A. A.; Hubbard, R. E.; Scott, R. K.; Williams, D. H.; J. Mol. Biol. 1999, 285, 713.

46. Bertrand, J. A.; Thieffine, S.; Vulpetti, A.; Cristiani, C.; Valsasina, B.; Knapp, S.; Kalisz, H. M.; Flocco, M.; J. Mol. Biol. 2003, 333, 393.

47. Leclerc, S.; Garnier, M.; Hoessel, R.; Marko, D.; Bibb, J. A.; Snyder, G. L.; Greengard, P.; Biernat, J.; Wu, Y.; Mandelkow, E.; Eisenbrand, G.; Meijer, L.; J. Biol. Chem. 2001, 276, 251.

Submitted: May 21, 2019 Published online: August 29, 2019 